\title{
Spatial DNS of an Isothermal Flat Plate Supersonic Turbulent Boundary Layer with/out Impinging Shock Wave*
}

\author{
Yusuke TOKURA** and Hiroshi MAEKWA** \\ ${ }^{* *}$ Department of Mechanical Engineering and Intelligent Systems, The University of \\ Electro-Communications \\ 1-5-1 Chofugaoka, Chofu-shi, Tokyo 182-8585, Japan \\ E-mail: maekawa@mec.uec.ac.jp
}

\begin{abstract}
Spatial direct numerical simulation results are presented for transitional/turbulent supersonic isothermal flat plate boundary layers at $M=2.0$ and impinging shock wave-boundary layer interactions. The numerical results show the formation and development of three-dimensional vortical structures such as hairpin packets and streak-breakdown, and secondary and tertiary hairpins as well. These characteristic vortical structures create a significant fraction of the supersonic turbulent boundary layer structure. An incident shock wave impinging upon the transitional boundary layer with streaks and hairpins, and unsteady reflected waves from the mildly separation boundary layer are observed. Expansion waves after the impinging point and compression waves due to the boundary layer reattachment are also identified. Across the interacting shock, turbulence is enhanced with finer hairpins generated inside, and it undergoes a relaxation process to higher Reynolds turbulent boundary layers far downstream.
\end{abstract}

Key words: DNS, Supersonic Turbulent Boundary Layer, Shock-Boundary Layer Interactions

\section{Introduction}

Physical phenomena associated with turbulent boundary layers and shock waves are commonly observed in aerospace applications and are important to the development of high-speed vehicles and propulsion systems. Unsteady shock/boundary layer interactions are of extreme relevance for aerospace application design, such as aircraft performance, structural fatigue, and air intake efficiency due to boundary layer separation.

The physical understanding of transition to turbulence due to various effects, free stream turbulence, surface roughness, and wall thermal conditions etc. is also crucial to modeling of transitional flow and effective to design various flows of practical interest, such as high-speed wings, turbo-machines, and launch vehicles during the ascent phase.

The interaction of shock waves with turbulent boundary layers is the subject of extensive research for its relevance in transonic, supersonic and hypersonic flows. A fundamental understanding of the complex physical phenomena is important to improve engineering models and to attempt to control their detrimental effects. The shock wave/boundary layer interaction is either produced at the surface where the boundary layer is developing, or where the shock wave impinges on the boundary layer, spatially evolving transitional or turbulent flows, being generated from an external source. Accordingly, shock wave /boundary layer interactions are classified as compression corner interactions or incident shock interactions. In the former case the body of experimental results is

Received 3 Sep., 2010 (No. 10-0395) [DOI: 10.1299/fst.6.30]

Copyright $\odot 2011$ by JSME 
comparatively much larger than the latter case. The two types of interactions have similar features: for example, the boundary layer develops under adverse pressure gradient; turbulence is enhanced across the interacting shock, and it undergoes a relaxation process downstream of the interaction.

Significant advances in the understanding of shock-wave/boundary layer interactions have been made in the past few decades, even though turbulence amplification mechanisms are not yet well understood. On the other hand, the progress of transitional scenarios and a characterization of structures of inhomogeneous turbulence and of its properties in the supersonic and hypersonic regime has not been as significant due to intrinsic difficulties both in the experiments and in the numerical simulations. The experimental measurements are limited to basic turbulence quantities and by the spatial resolution close to the wall, among other difficulties. The simulations to mimic the experimental study of transition to turbulence have been hampered by large cost.

Guarini et al. ${ }^{(1)}$ investigated the applicability of Morkovin's hypothesis and the Strong Reynolds Analogy (SRA) by using an extended temporal turbulent boundary layer simulation for Mach 2.5. They found that an extended form of the Reynolds analogy holds. Gatski et al. ${ }^{(2)}$ and Pirozzoli et al. ${ }^{(3)}$ performed an adiabatic flat plate spatial DNS for Mach 2.25 to investigate the turbulent kinetic energy budget. The rate of turbulence production was found to be larger than for the incompressible case, as shown by Guarini et al. ${ }^{(1)}$, but the turbulent kinetic energy budget shows similarity with the incompressible case provided all terms are properly scaled. A region of blowing and suction is introduced to induce transition, however, the connection to the streak breakdown and further to turbulence is not addressed in the DNS study of Pirozzoli et al. ${ }^{(3)}$

Adams $^{(4)}$ has performed the first direct numerical simulation of a shock wave interacting with a turbulent boundary layer developing on a compression ramp at free stream Mach number $M=3$. The author used a fifth-order hybrid compact finite difference/ENO scheme for the spatial discretization of the convective fluxes, and six-order central compact finite differences for the viscous fluxes. Adams observed a small separated flow in the proximity of the flat plate/ramp junction and showed that the strong Reynolds analogy does not hold in the interaction zone. Pirozzoli and Grasso ${ }^{(5)}$ have carried out a DNS study of an oblique shock wave impinging upon a turbulent boundary layer at conditions that mimic the experimental conditions by Deleuze ${ }^{(6)}$. They found that the incoming boundary layer undergoes mild separation and coherent structures are shed near the separation point. Their simulation showed the occurrence of low-frequency tones in the interaction zone. They proposed a resonance mechanism responsible for the large-scale low-frequency unsteadiness similar to the generation of tones in cavity flows.

In the present study, a spatial DNS of a supersonic isothermal flat plate boundary layer flow at Mach 2.0 is analyzed. Effects of both transition and boundary layer growth require the application of the fully spatial formulation in a direct numerical simulation without extended temporal simplifying assumptions. The emphasis of this study is to assess the transitional scenarios for supersonic boundary layer flow, the late stage of streak breakdown and also fully turbulent boundary layer quantities developing on an isothermal flat plate. Furthermore, we consider the problem of a shock impinging upon a transitional boundary layer. Although less studied experimentally, we feel that the shock impinging is appropriate to understand the complicating effects of bulk flow compression due to the shock that contributes significantly to turbulence amplification and formation of large-scale structures.

\section{Numerical Method}

In the DNS of spatially developing boundary layers, the non-dimensional equations governing the conservation of mass, momentum, and energy for a compressible Newtonian fluid are solved. Note that displacement thickness at the inlet boundary layer $\left(\delta^{*}\right)$ is chosen 
to be a representative length scale.

$$
\begin{aligned}
& \frac{\partial \rho}{\partial t}+\frac{\partial\left(\rho u_{i}\right)}{\partial x_{i}}=0 \\
& \frac{\partial\left(\rho u_{i}\right)}{\partial t}+\frac{\partial\left(\rho u_{i} u_{j}\right)}{\partial x_{j}}=-\frac{\partial p}{\partial x_{i}}+\frac{\partial \tau_{i j}}{\partial x_{j}} \\
& \frac{\partial E_{t}}{\partial t}+\frac{\partial\left(E_{t} u_{j}\right)}{\partial x_{j}}=-\frac{\partial\left(p u_{j}\right)}{\partial x_{j}}+\frac{\partial\left(u_{i} \tau_{i j}\right)}{\partial x_{j}}-\frac{\partial q_{j}}{\partial x_{j}}
\end{aligned}
$$

Assuming constant specific heats, the perfect gas equation of state is written as

$$
p=\frac{1}{\gamma M^{2}} \rho T .
$$

The components of the viscous stress tensor $\tau_{i j}$ are

$$
\tau_{i j}=\frac{\mu}{\operatorname{Re}}\left[\left(\frac{\partial u_{i}}{\partial x_{j}}+\frac{\partial u_{j}}{\partial x_{i}}\right)-\frac{2}{3} \delta_{i j} \frac{\partial u_{k}}{\partial x_{k}}\right]
$$

where $R e$ is the Reynolds number based on the displacement thickness at the inlet boundary layer and the free stream velocity. The non-dimensional, temperature dependent dynamic viscosity is approximated by a power law as

$$
\mu=T^{\frac{2}{3}}
$$

By assuming a unity Prandtl number $\left(P_{r}=1.0\right)$ and constant ratio of the specific heats $(\gamma=$ 1.4), the thermal conductivity $k$ is proportional to the viscosity expressed by

$$
k=\frac{\mu}{(\gamma-1) M^{2} \operatorname{Re} \operatorname{Pr}}
$$

and the heat flux vector is given by

$$
q_{i}=-k \frac{\partial T}{\partial x_{i}}
$$

To obtain spatially accurate numerical solutions to the governing equation, high-order(6th-order and 5th-order) compact schemes (Lele ${ }^{(7)}$ and Deng, Maekawa, and Shen ${ }^{(8)}$ ) are employed. The compact 5 th-order up-wind biased scheme is used to discretize the hyperbolic terms, the compact 6th-order scheme for the viscous terms. A 4th-order Runge-Kutta method is employed for the time integration. In the present simulation, $\operatorname{NSCBC}^{(9)}$ (Navier-Stokes Characteristic Boundary Conditions) are used in the streamwise $(x)$ and vertical $(y)$ directions, and periodic boundary conditions in the spanwise $(z)$ direction. A no-slip adiabatic/isothermal wall condition and non-reflecting boundary conditions are imposed at $y=0$ and upper far field, respectively. The length of the computational domain in the spanwise direction is determined from the previous turbulent boundary layer temporal simulations (Guarini et al. ${ }^{(1)}$ ). The optimization of the up-wind biased scheme, which improves the efficiency of the resolution and confines the dissipative effects to higher wavenumbers, is helpful to remove the high wavenumber errors and 
preserve high resolution for the low and intermediate wavenumbers. The spectral-like resolution characteristics of the optimized 5th-order upwind biased scheme are shown in Fig.1 with plots of the modified wavenumber against wavenumber $k^{*}$ of Fourier coefficients, where the resolution characteristics of different schemes such as 6th-order and optimized 9th-order schemes can be compared. Numerical tests ${ }^{(10)}$ for various compressible flows, such as strong shock-vortex interactions and acoustics, and unstable compressible free shear flows, have been performed with a few linear compact schemes. The dissipative effect confined to high wave numbers has an advantage to avoid contamination by spurious point-to-point oscillations due to the shock.

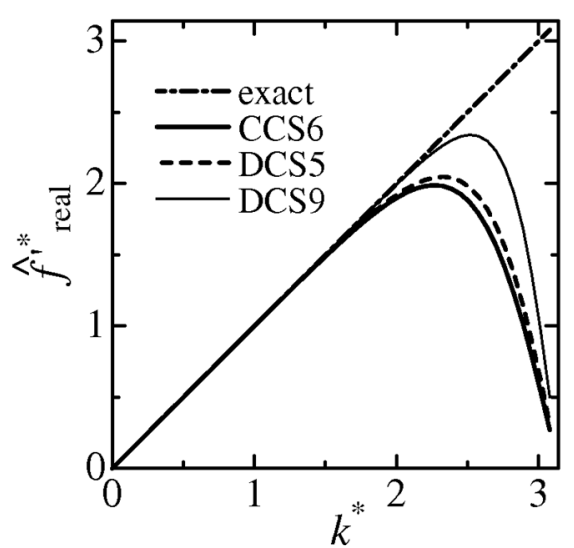

(a)

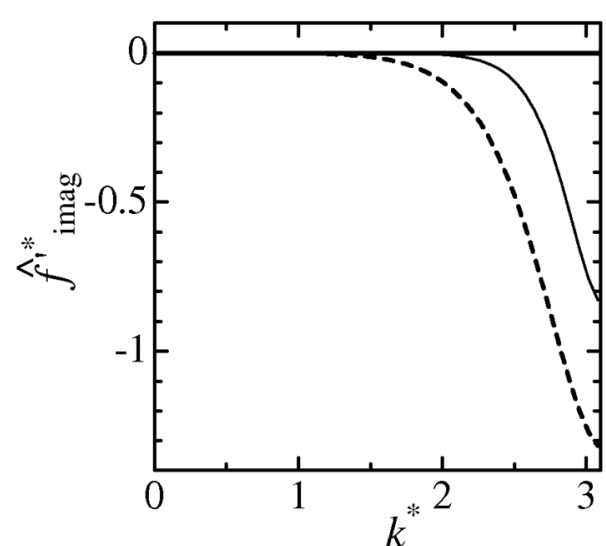

(b)

Fig. 1 Fourier analysis of differencing errors: (a) modified wavenumber vs wavenumber for first derivative approximation and (b) imaginary part of modified wavenumber. Exact, CCS6, DCS5 and DCS9 indicate spectral method, 6th-order central compact, 5th-order upwind compact, and 9th-order compact schemes, respectively.

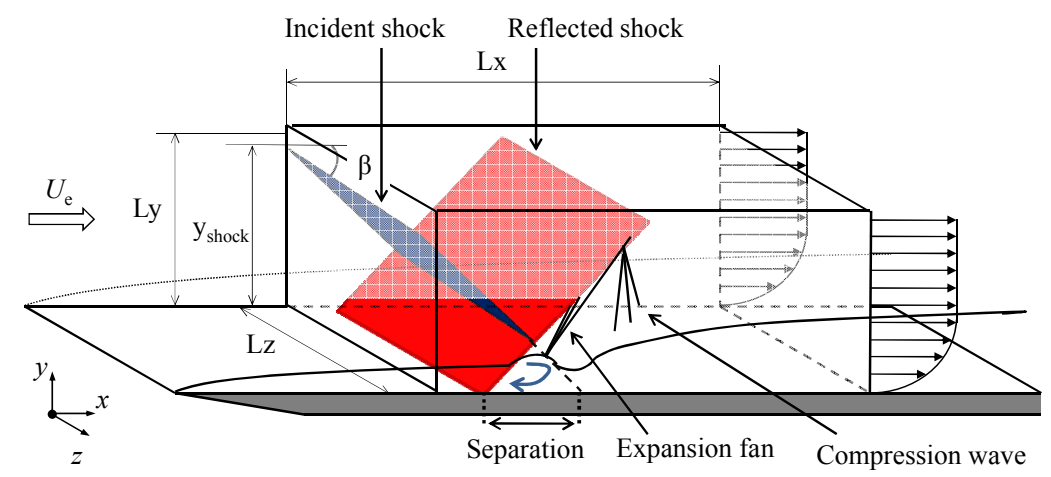

Fig. 2 Sketch of the computational domain and Cartesian coordinates.

High amplitude (5\%) disturbances of the free stream velocity are superimposed on the laminar velocity profile. Isotropic turbulence is used to generate the inflow disturbance, that has a spectrum energy of the type $E(k)=k^{4} \exp \left[-2\left(k / k_{0}\right)^{2}\right]$ where $k$ is wave number and the peak of the spectrum is located at $k_{0}$. Figure 2 shows a sketch of the computational domain and Cartesian coordinates. An extensive grid sensitivity analysis ensures grid independency. For the no-shock case, the grid resolutions are reported with main parameters given in wall units in Table 1, where $\Delta y_{w}$ indicates a minimum spacing in the wall normal direction. Assessment of the domain and resolution size is made by comparison to the DNS of Guarini et al. ${ }^{(1)}$ at the equivalent locations in the downstream turbulent boundary layer. An oblique 
shock wave, whose intensity in terms of pressure ratio is 1.25 , is made to impinging on the transitional boundary layer forced by the inflow disturbances as stated above developing on the isothermal flat plate at $M=2.0$. The flow-field is initialized using the numerical solution of the isothermal no-shock case. The shock is artificially generated by enforcing at the inlet boundary conditions that satisfy the Rankine-Hugoniot relations. For the shock case, the grid resolutions will be given at the location where downstream turbulent boundary layer reaches an equilibrium state.

\begin{tabular}{|c|c|c|c|c|c|}
\hline \multicolumn{7}{|c|}{ Table 1 Grid parameters } \\
\hline \hline & $M$ & $R e_{\theta}$ & $N x \times N y \times N z$ & $L x^{+} \times L y^{+} \times L z^{+}$ & $\Delta x^{+} \times \Delta y_{w}{ }^{+} \times \Delta z^{+}$ \\
\hline Present & 2.0 & 960 & $751 \times 241 \times 120$ & $11554^{+} \times 681^{+} \times 800^{+}$ & $15.5^{+} \times 0.89^{+} \times 6.72^{+}$ \\
\hline Guarini $^{(1)}$ & 2.5 & 1577 & $256 \times 209 \times 192$ & $2269^{+} \times 875^{+} \times 1132^{+}$ & $8.96^{+} \times 0.48^{+} \times 5.91^{+}$ \\
\hline
\end{tabular}

\section{Results}

\subsection{Isothermal flat plate transitional/turbulent boundary layer}

Figures $3 \mathrm{a}$ and $\mathrm{b}$ shows the visualized low-speed streaks and quasi-streamwise vortices with hairpin packets. In this study, a spatial transitional DNS of an isothermal flat plate boundary layer flow at Mach number 2.0 is analyzed. The Reynolds number at the inlet is 1000 , based on the displacement thickness. The disturbances are introduced both inside and outside the boundary layer $\left(y / \delta *_{0}<10.0\right)$. The grid and computational domain sizes are $N x \times N y \times N z=751 \times 241 \times 120$ and $L x \times L y \times L z=260 \delta^{*}{ }_{0} \times 15 \delta^{*}{ }_{0} \times 18 \delta^{*}{ }_{0}$, respectively. The quasi-streamwise vortices are visualized by the second invariant of the velocity gradient tensor $Q$.

This figure indicates the transition scenario of the following stages. First, the generation of quasi-streamwise vortices gives rise to the low-speed streaks $(t=107.5)$ and the vortices elongate when they age. These vortices induce the lift-up of the downstream streaks ( $t=$ 107.5). Secondly, a hairpin head appears on the lifted low-speed streak leads to the formation of pairs of counter-rotating leg vortices and then hairpins occur in streamwise succession $(t=120.0)$. More upward lift-up of the low-speed streak yields streak valleys ${ }^{(11)}$ downstream that populate hairpins leading to a spot-like structure $(t=135.0)$. The successive vortices around the streak valley due to their interaction lift up the low-speed streaks and elongate them. This process is enhanced during the downstream motion $(t=160.0)$. The elongated lift-up low-speed streak oscillates while traveling downstream due to its instability, and it induces new arch vortices on the streak and generates vortices that are aligned largely in the streamwise direction (hairpin packets $\left.{ }^{(12)}\right)(t=180.0)$. By the formation of an arch vortex (horseshoe vortex ${ }^{(13)}$ ) spanning a few streaks, the elongated streak disappears. The upstream lift-up streak and adjacent streaks create other hairpin packets (secondary and tertiary hairpins). These later stage observations are similar to those in an adiabatic flat plate boundary layer ${ }^{(14)}$.

The low-speed streaks, the valleys, watersheds and hairpin packets are observed for both cases (Figs. 4, 5). In the DNS study by Toh and Itano ${ }^{(11)}$, the branches of the low-speed streaks are reminiscent of rivers in a map of a mountainous area in the top view of the channel flow. They call the region where the branches gather "valley" and the region where the branches emerge "watershed". They suggest that when two streak structures near the wall merge, a concentrated eruption occurs which causes an influx of fluid from a near-wall region into the outer region. Careful examination indicates that more low-speed streak valleys and watersheds are generated in the isothermal case than in the adiabatic case (Fig. 4). This suggests that the isothermal low wall temperature condition with large disturbances enhances the evolution of higher streamwise vortices closer to the wall, from which results 
Science and Technology
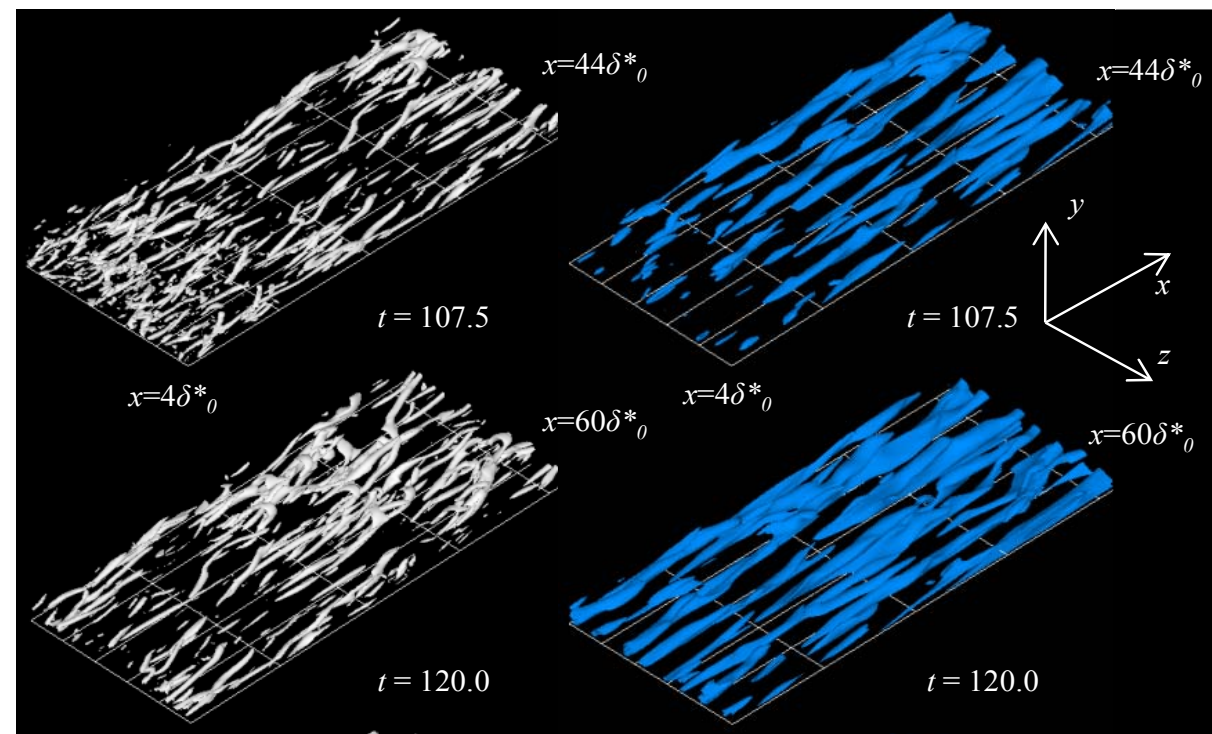

$x=20 \delta *_{0}$

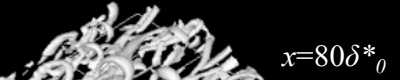
$3-3.2,314$ ?

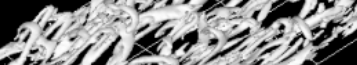

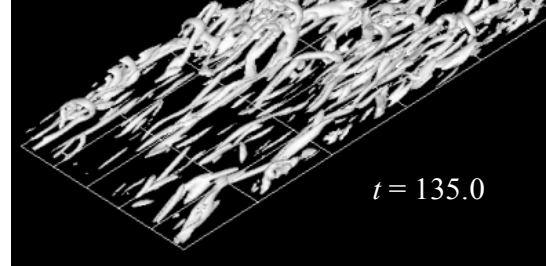

Watershed

$=20 \delta^{*}{ }_{0}$
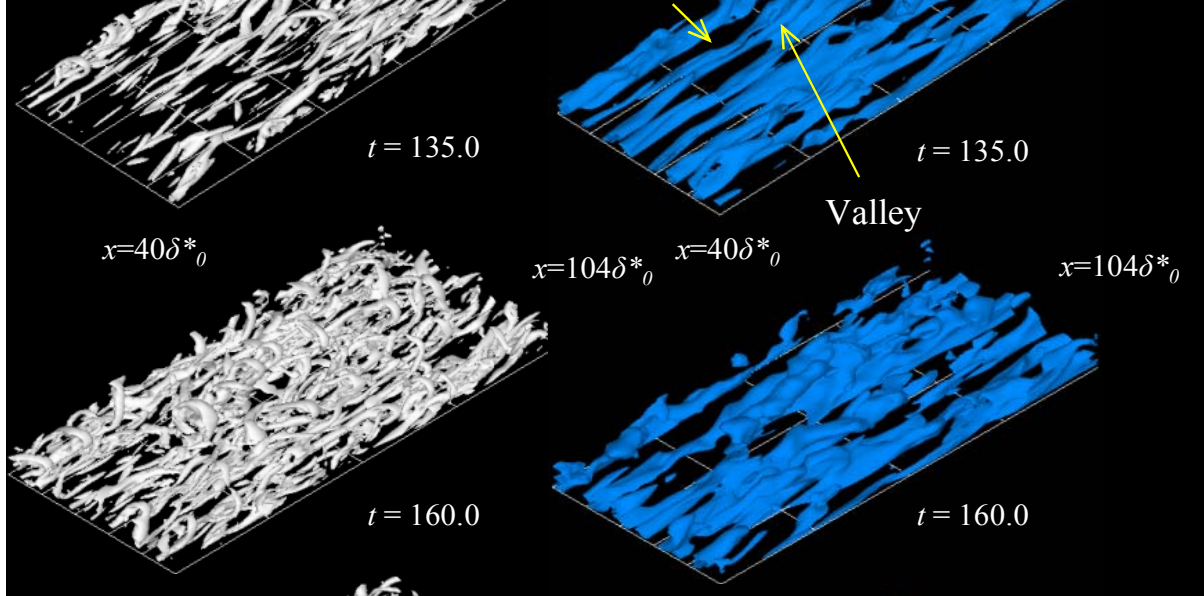

$x=64 \delta^{*}$
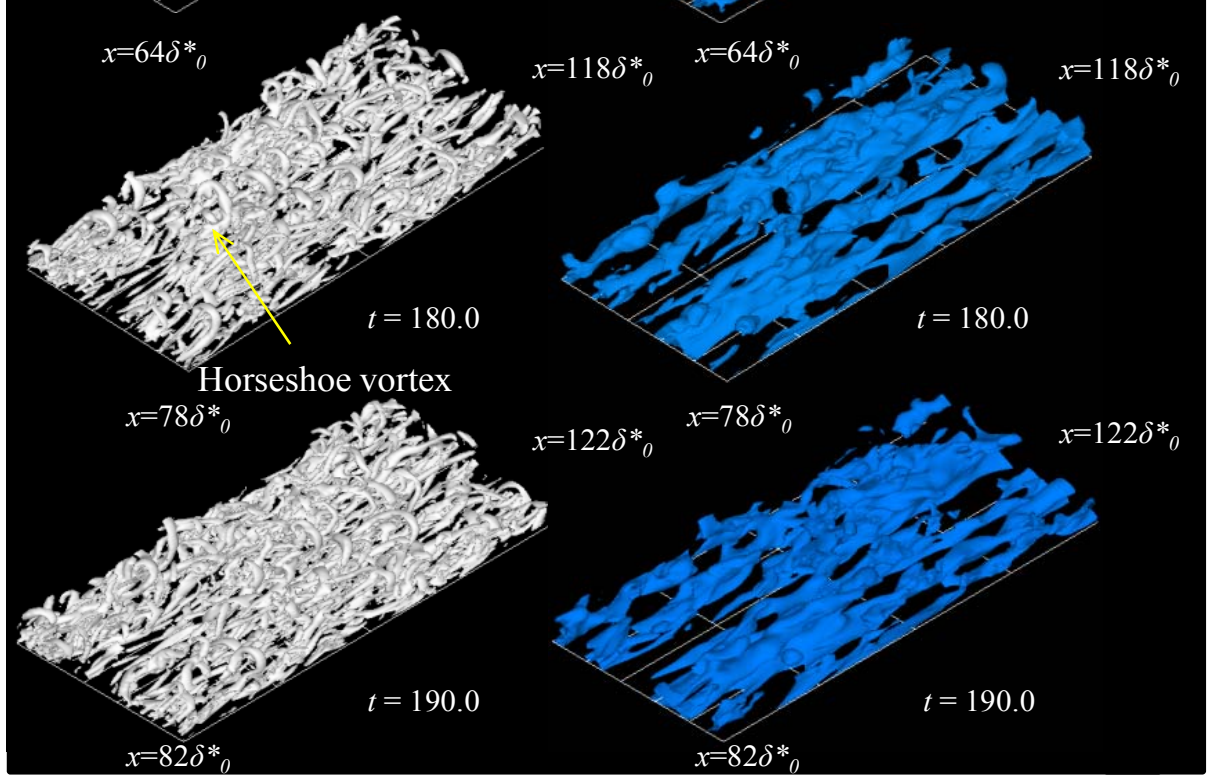

(a) Vortex structures

(b) Low-speed streaks

Fig. 3 Low-speed streak structures $\left(u^{\prime}=-0.1\right)$ and packet of hairpins $(Q=0.03)$. 


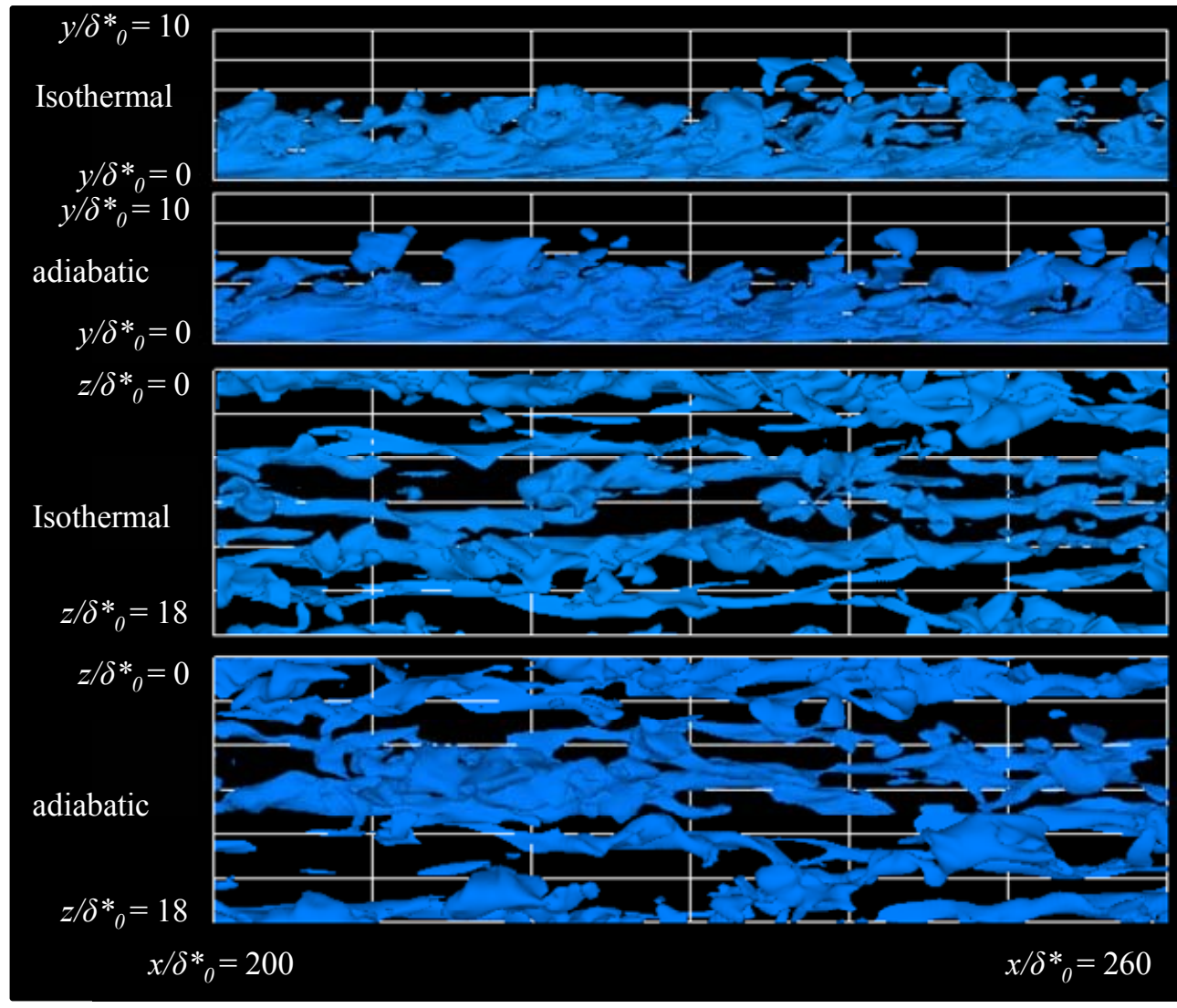

Fig. 4 Low-speed streak structures $\left(u^{\prime}=-0.1\right)$.

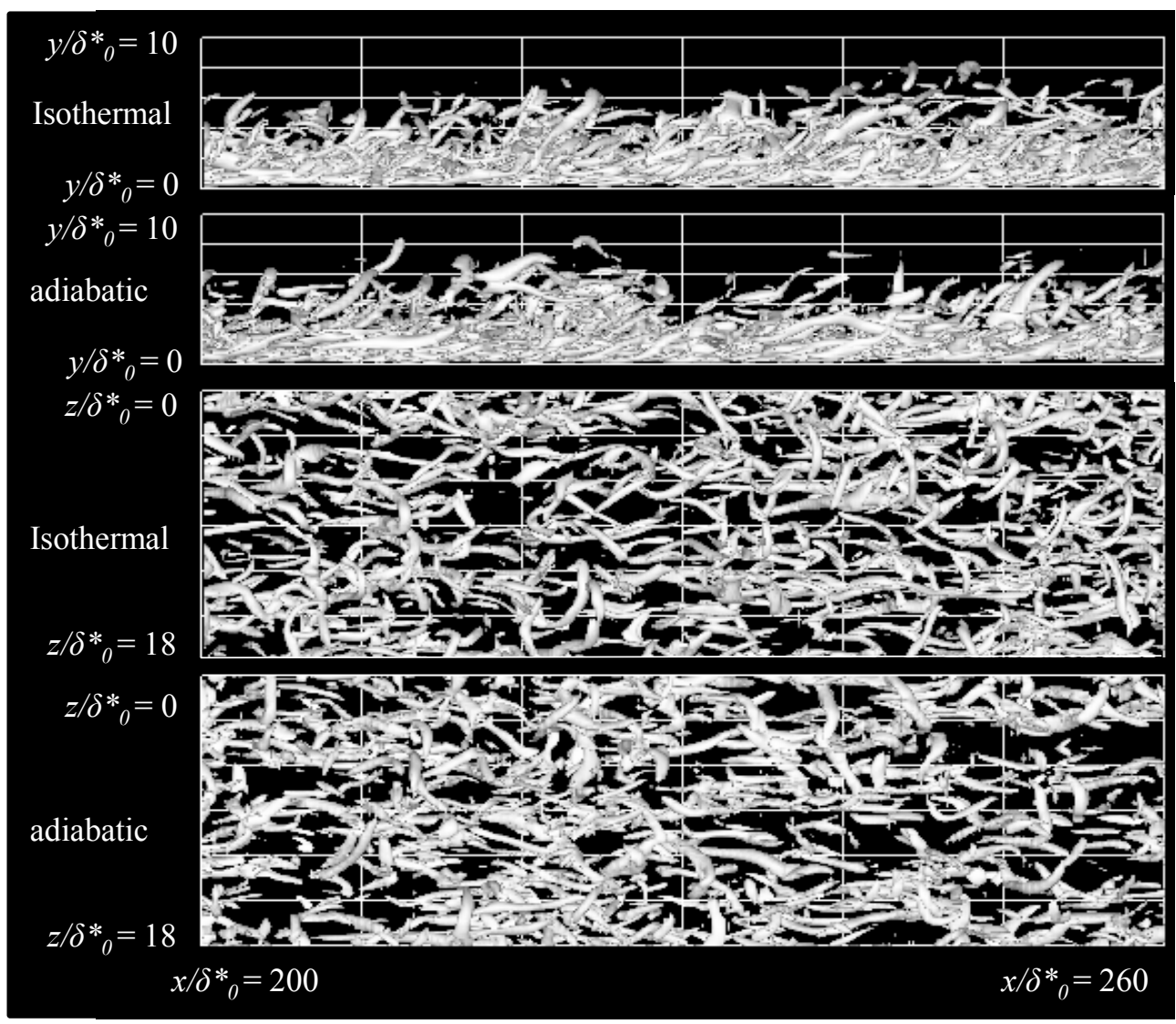

Fig. 5 Vortex structures $(Q=0.03)$. 
the development of populated low-speed streaks due to the downstream integration effects of the smaller viscosity near the wall.

Figure 6 shows the velocity profile at the locations of $\operatorname{Re}_{\theta}=960\left(\operatorname{Re}_{\theta}=990\right.$ for the adiabatic case). The Van-Driest transformed velocity $u_{v d}$ is plotted in wall units in Fig. 6. $u_{v d}$ is defined as

$$
u_{v d}=\int_{0}^{u^{+}}\left(\frac{\rho}{\rho_{w}}\right)^{\frac{1}{2}} d u^{+}
$$

Here, $u^{+}=u / u_{\tau}$, and $\rho_{w}$ is the density at the wall, where $u_{\tau}$ is the friction velocity. On the plot we have included the linear sub-layer relation $u^{+}=y^{+}$, and the standard log-law with constants $\kappa=0.41, C=5.0$ for the plot. Figure 6 shows that in the region of $20<y^{+}<80$, the simulation data fall on the log-law curve.

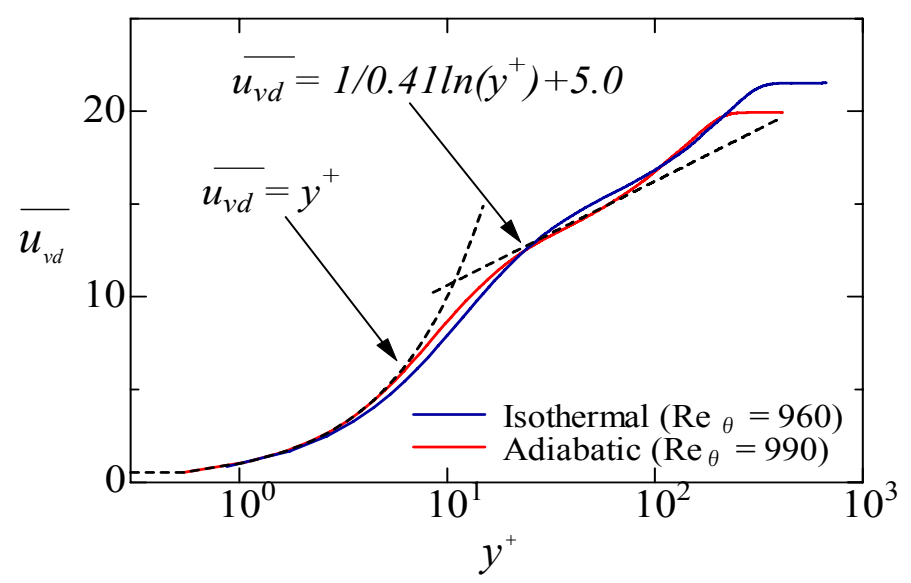

Fig. 6 Van-Driest transformed velocity in wall units.

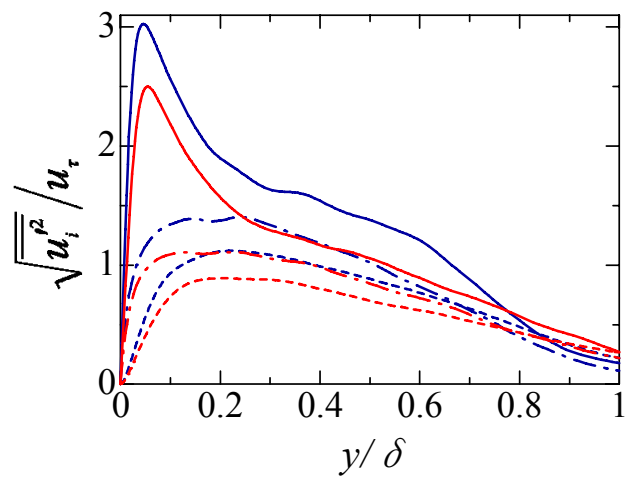

(a)

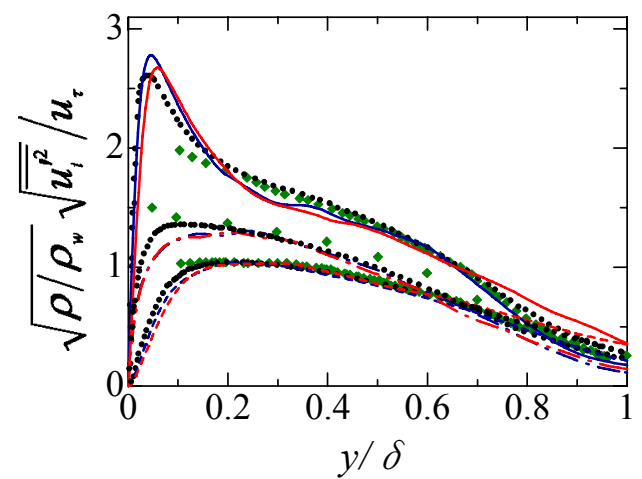

(b)

Fig. 7 R.M.S. velocity profiles plotted versus $y / \delta$, (a) scaled by $1 / u_{\tau}$, (b) scaled by $\sqrt{ }\left(\rho / \rho_{w}\right) \times 1 / u_{\tau}$. Blue lines correspond to the isothermal case, red lines correspond to the adiabatic case and symbols are used for the data by Spalart and Klebanoff:___, streamwise velocity component; - - - , wall-normal velocity component; -...-., spanwise velocity component; black circles, Spalart $\left(\operatorname{Re}_{\theta}=670\right)$; green diamonds, Klebanoff $\left(R e_{\theta}=7750\right)$.

Figures $7 \mathrm{a}$ and $\mathrm{b}$ show the R.M.S. velocity profile. When the data are scaled by the square root of the mean density as proposed by Morkovin ${ }^{(15)}$ (1962), there is good 
agreement for all three velocity components in the upper region from the wall, but near the wall, the isothermal case's R.M.S. data are larger than those in the adiabatic case due to the difference of the density profile (Fig. 7b). This figure shows that the maximum of the streamwise component shifts toward the wall in the isothermal case. In addition, the incompressible DNS data of Spalart ${ }^{(16)}$ for $\operatorname{Re}_{\theta}=670$ with fine resolutions and the subsonic experimental study of Klebanoff ${ }^{(17)}$ are presented in Fig. $7 \mathrm{~b}$. The present data follow closely the numerical data of Spalart ${ }^{(16)}$ and those of Klebanoff ${ }^{(17)}$ away from the wall.

\subsection{Shock impinging on the transitional boundary layer}

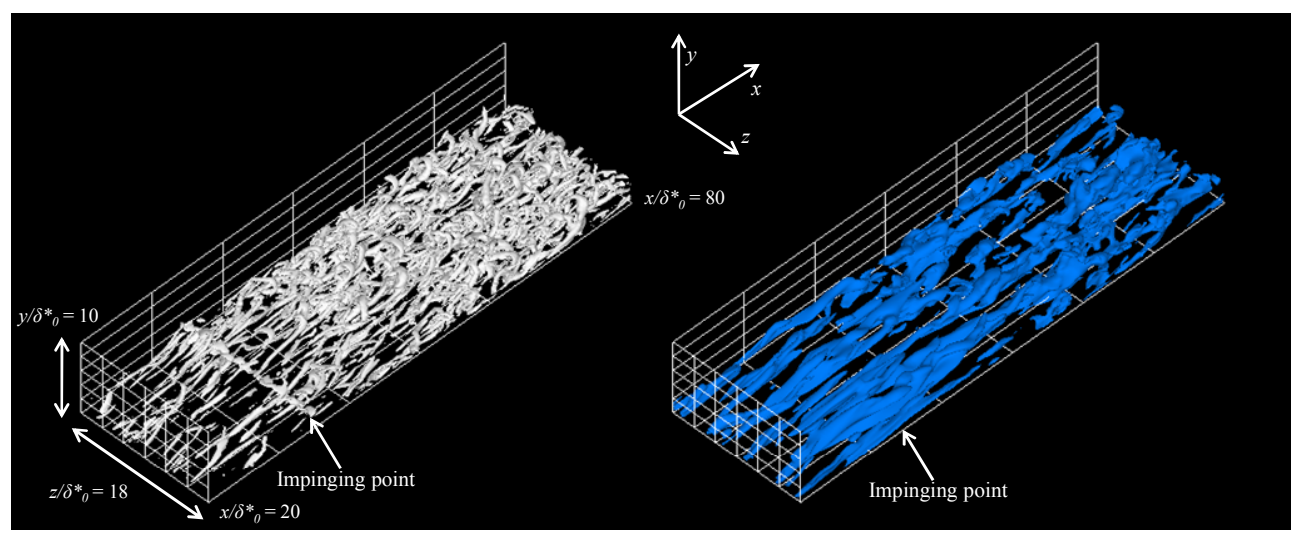

Fig. 8 Vortex and Low-speed streak structures.

(a)

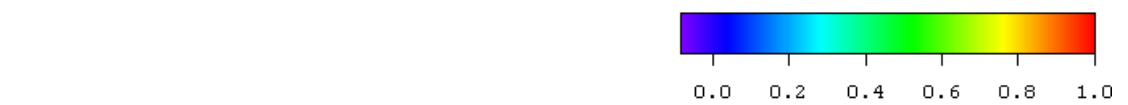

(b)

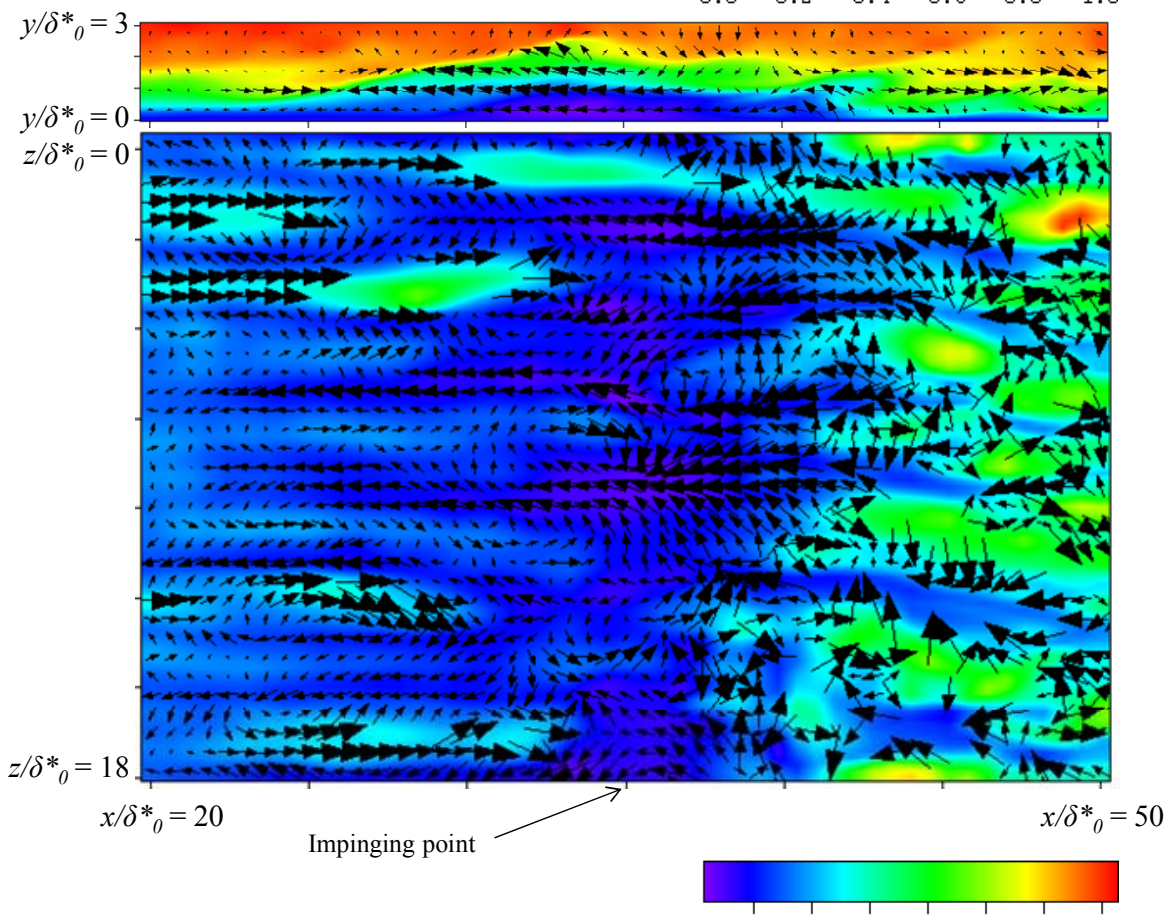

$\begin{array}{lllllll}0.0 & 0.1 & 0.2 & 0.3 & 0.4 & 0.5 & 0.6\end{array}$

Fig. 9 The instantaneous velocity vectors. (a) Velocity vectors in a plane vertical to the wall $\left(z / \delta^{*}{ }_{0}=8.0\right)$, where the velocity vectors represent the streamwise perturbation velocity $\mathrm{u}^{\prime}$ and wall-normal velocity $v$. (b) Velocity vectors in a plane parallel to the wall $\left(y / \delta^{*}{ }_{0}=0.1\right)$, where the velocity vectors represent the streamwise perturbation velocity $u^{\prime}$ and spanwise velocity $w$. 
In order to understand some of the dynamic features of the shock impinging flow field, we analyze the instantaneous and mean flow properties, and the turbulence statistics from the simulation. For the shock impinging simulation, the computational domain size and the grid resolution are $L x^{+} \times L y^{+} \times L z^{+}=117628^{+} \times 1762^{+} \times 1057^{+}, \Delta x^{+} \times \Delta y_{w}{ }^{+} \times \Delta z^{+}=23.5^{+} \times 0.86^{+} \times 8.88^{+}$, respectively, which is comparable or better than the $\mathrm{DNS}^{(18)}$ by $\mathrm{Li}$ and Coleman of the shock impinging simulation with the grid resolution of $\Delta x^{+} \times \Delta y_{w}{ }^{+} \times \Delta z^{+}=19^{+} \times 1.0^{+} \times 12^{+}$.

\section{A. Instantaneous flow properties}

The general features of the flow are represented in terms of the instantaneous flow properties, such as the second invariant of the velocity gradient tensor $Q$, the low-speed streak, pressure gradient and dilatation fields, and fluctuation velocity vectors. The instantaneous $Q$ and the low-speed streak structures reveal the inherent vortical structure at the impinging line, which is different from the ordinary turbulent boundary layer with hairpins. A comparison of Fig. 8 and Fig. $3 \mathrm{~b}(t=107.5)$ indicates that the separated region shown in Fig. 9 has a streaky structure, where more quasi-streamwise and hairpin vortices are generated. Figure 9a of the instantaneous velocity vectors in a plane vertical to the wall shows the boundary layer undergoes mild separation and reattaches downstream, where the streamwise velocity fluctuations are produced by subtracting the averaged velocity from the instantaneous velocity. As will be described, the mean flow properties indicate that flow separation occurs around the impinging point. The instantaneous velocity vectors around the impinging point in Fig. 9b show that flow separation occurs three dimensionally involving backward and forward flows. This simulation with an unsteady small separation flow shows

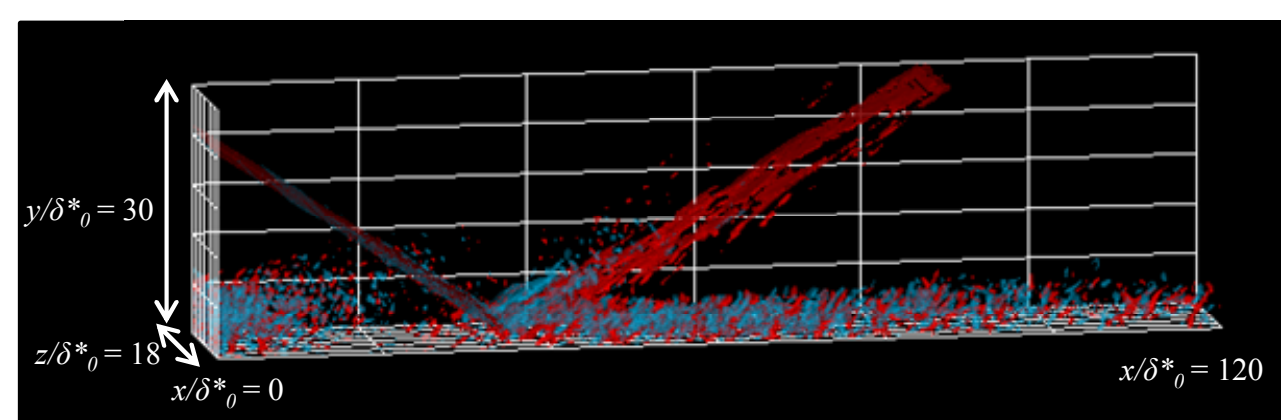

Pressure gradient $(\partial p / \partial x= \pm 0.02)$

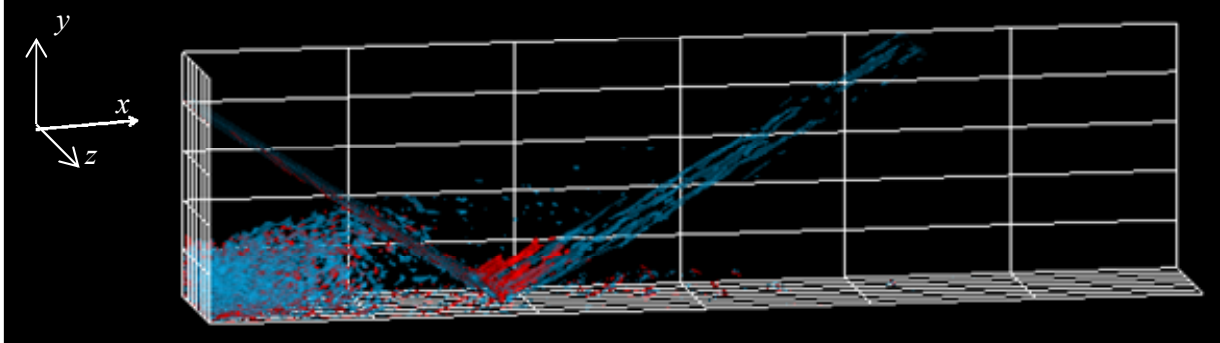

Dilatation field ( $\operatorname{div} u= \pm 0.075)$

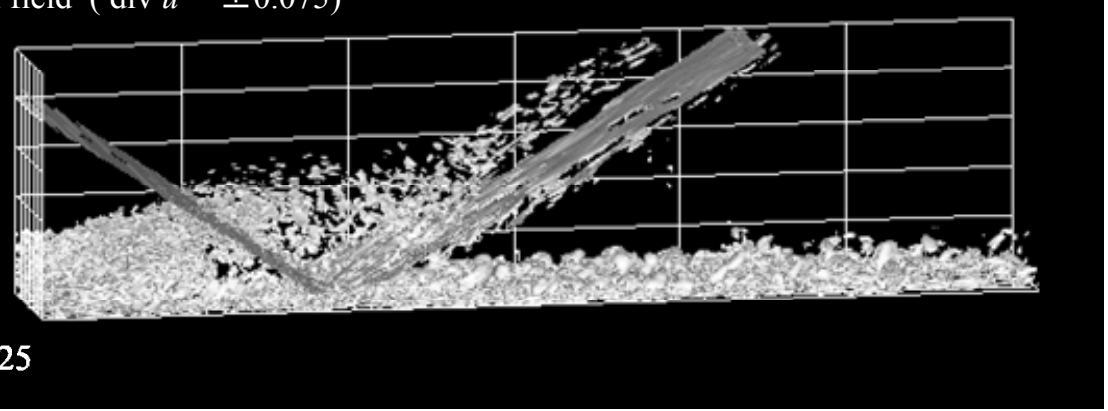

Fig. 10 The pressure gradient, dilatation, and $|\nabla p|$ fields. 


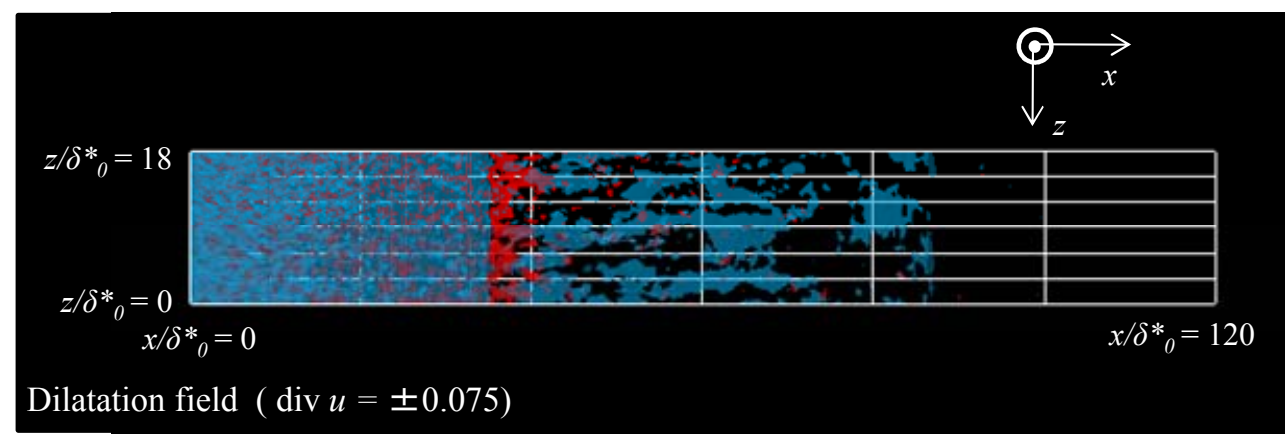

Fig. 11 The dilatation field (Top view).

that the turbulence enhancement is primarily associated with the adverse pressure gradient and a direct effect of the shock, which is different from the amplification mechanism in the DNS with the mixing layer by Pirozzoli and Grasso ${ }^{(5)}$. Andreopoulos et al. ${ }^{(19)}$ have analyzed several types of shock/turbulence interactions and conclude that turbulence amplification through shock wave interaction is a direct effect of the Rankine-Hugouniot relations.

Figures 10 and 11 present the instantaneous shock system around the impinging point using the pressure gradient and dilatation fields. The instantaneous pressure gradient and the corresponding dilatation field indicate that the incident shock is characterized by a compression zone (blue in the figure) and a steep pressure gradient along the $x$ direction and that an unsteady expansion wave fan is found after the two-dimensional impinging line where positive dilatation (red region in the dilatation field) are observed and the pressure gradient along the $x$ direction is mildly favorable (red region in the figure). Downstream of the expansion fan, unsteady compression waves are observed, where a compression zone and an adverse pressure gradient region are found. Figure 11 supports these findings of the incident shock, expansion fan waves, and reflected compression waves from the top view. The instantaneous $|\nabla p|$ presents well the incident shock and the unsteady reflected compression waves. The unsteady expansion fan and compression waves are attributed to the interaction of unsteady large-scale motions with the impinging shock. The unsteady reflected shock due to the boundary separation upstream the compression waves are seen far away from the boundary layer. As will be discussed in the mean flow properties subsection, the reflected shock will be observed due to the boundary layer separation. However, the instantaneous dilatation field is so weak that the reflected waves are hardly seen upstream of the impinging point.

\section{B. Mean flow properties}

Figure 12 shows the contours of the mean Mach number. The incoming transitional boundary layer interacts with the oblique shock wave at $x=35.6 \delta^{*}$, and the boundary separates mildly upstream, the compression wave generated at the separation point is very weak. The separated boundary layer reattaches downstream to form a reattachment compression wave.

The local friction coefficient is plotted in Figs. 13a and b. Figures 13a and b indicate the negative coefficient around the impinging point, where the mean separation point is $x=$ $32.4 \delta^{*}$ and the mean boundary layer reattaches at $x=37.6 \delta^{*}$. The friction coefficient downstream of the reattachment increases rapidly and reaches the turbulent boundary layer. Note the skin-friction coefficient fluctuation at around $x=120 \delta^{*}{ }_{0}$ is due to the effects of weakly reflected waves from the upper boundary, because this DNS does not have enough of a buffer zone where the compression waves from the impinging point are damped at the 


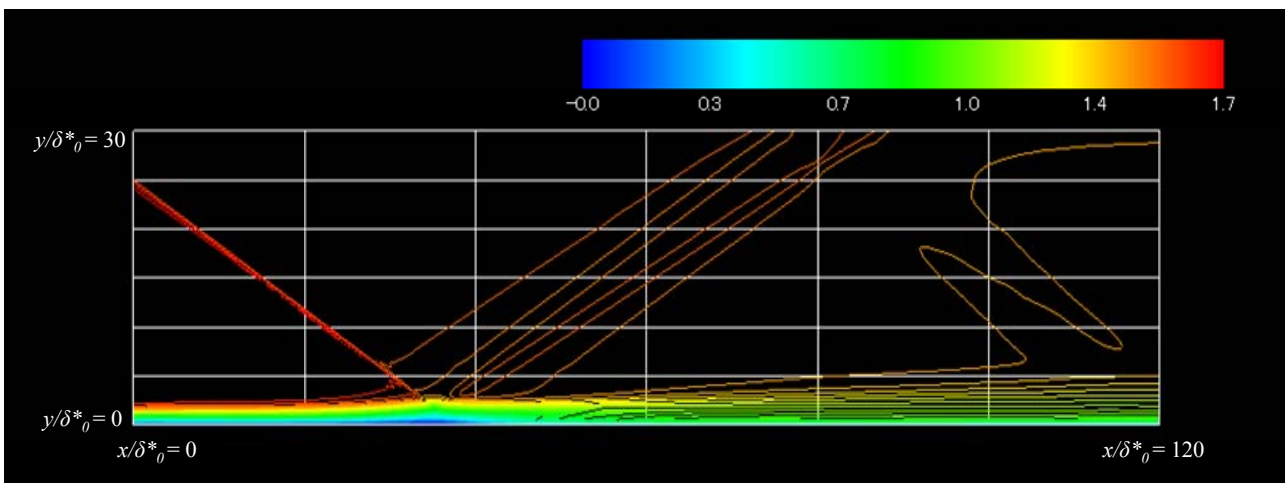

Fig. 12 Mean Mach number $(M)$ contours $(0.0<M<1.7$, Interval $(0.05))$.
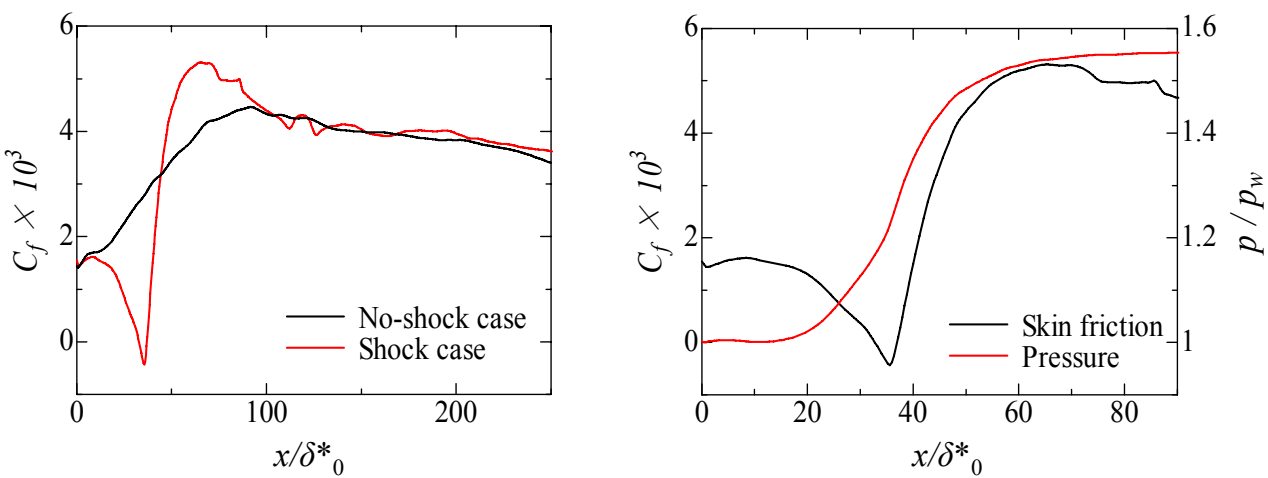

Fig. 13 Skin-friction coefficient and wall pressure distribution. (a) Comparison of the skin-friction coefficient with no-shock case and (b) Shock case skin-friction coefficient and pressure distribution upstream/downstream of the impinging point.

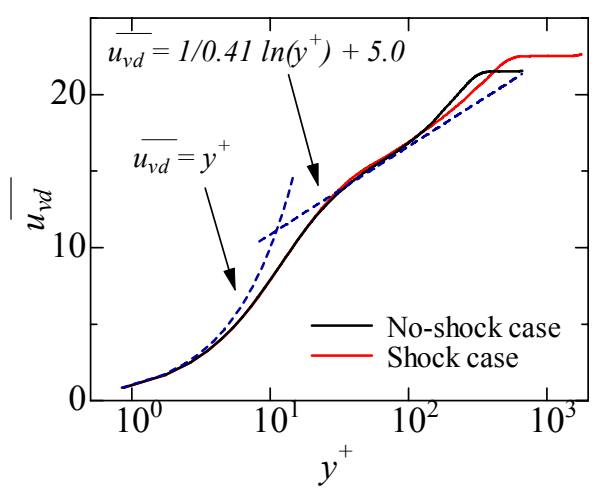

(a) Van-Driest transformed velocity in wall units

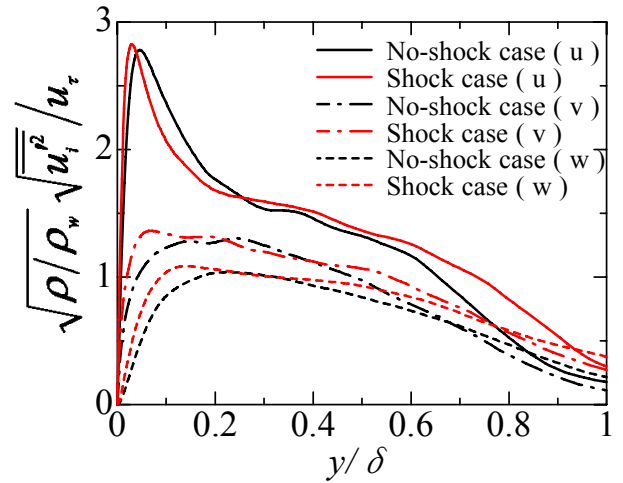

(b) R.M.S. velocity profiles

Fig. 14 Velocity profiles.

boundary.

As described both in the instantaneous flow and the mean flow sections, the simulation shows three distinct flow regions. First, a supersonic zero pressure gradient transitional boundary layer develops upstream of the interaction region. Secondly, the interaction between the impinging shock and boundary layer structures takes place in the interaction zone shown in Figs. 12 and 13b with a supersonic adverse pressure gradient region. Thirdly, a supersonic zero pressure gradient region appears downstream, where the turbulent flow relaxes to an equilibrium state. Figures $14 \mathrm{a}$ and $\mathrm{b}$ show the velocity profiles and R.M.S. 
velocity fluctuation data at the locations of $x=220 \delta^{*}{ }_{0}$ for both the no-shock case and the shock impinging case. The shock impinging case compares well with the no-shock case. This figure also shows that the maximum shifts toward the wall in the shock impinging case.

\section{Anisotropy of the Reynolds stress}

To characterize the change in anisotropy of the Reynolds stress tensor around the impinging point, we have focused on the invariants of the stress anisotropy tensor:

$$
b_{i j}=\frac{\overline{u_{i}} \overline{u_{j}}}{\overline{u_{k}}}-\frac{1}{3} \delta_{i j}
$$

and its scalar invariants:

$$
\Pi=b_{i j} b_{j i}, \quad \text { III }=b_{i j} b_{j k} b_{k i}
$$

As shown by Lumley ${ }^{(20)}$, all realizable fields must lie inside the anisotropy invariant map, whose vortices constitute special states of turbulence. The computed anisotropy invariant maps at various streamwise locations are depicted in Fig. 15. The triangle is the so-called Lumley triangle ${ }^{(20)}$.

Since the boundary separates mildly, the distribution is similar to one typical of canonical boundary layer flows at the point before the impinging point $\left(x=25 \delta_{0,}^{*} x=\right.$ $\left.32.4 \delta^{*}{ }_{0}\right)$. However in the point after the impinging point $\left(x=37.2 \delta^{*}{ }_{0}\right)$, the invariants, which lies along the two-component limit move towards the left corner point of the anisotropy map, which corresponding to the two-component axisymmetric state. In the point ( $x=$ $240 \delta_{0}^{*}$ ) where downstream turbulent boundary layer reaches an equilibrium state, the distribution converges to the canonical boundary layer flows.
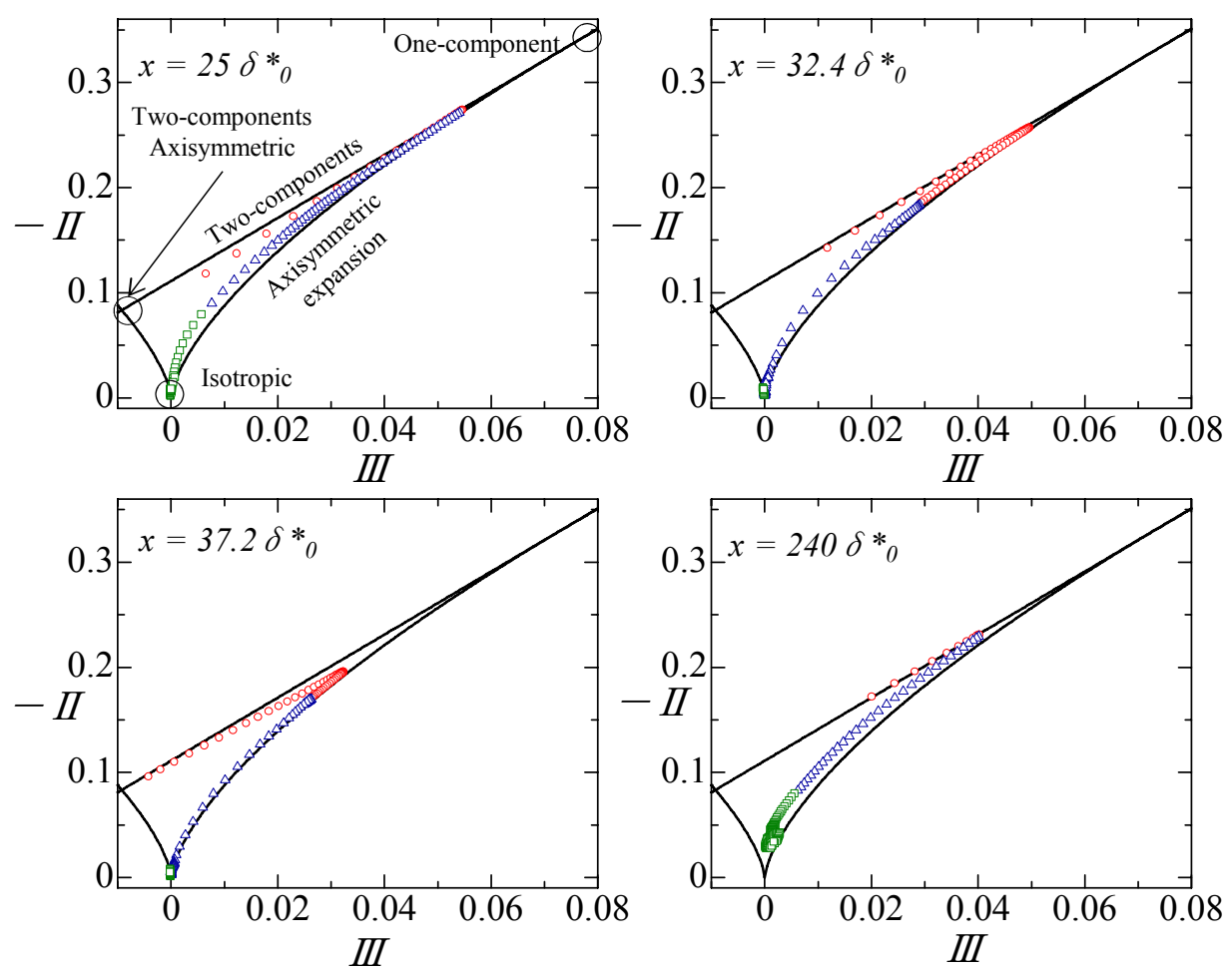

Fig. 15 Anisotropy invariant maps of the Reynolds stress tensor in the boundary layer at various streamwise locations. Red circles, $y+<10$; Blue triangles, $10<y+<50$; Green squares, $50<y+, y / \delta<1.0$. 


\section{Conclusions}

Direct numerical simulations of a Mach 2.0 spatially evolving transitional/turbulent boundary layer were carried out using high resolution spectral-like upwind biased compact schemes. The interaction of an impinging shock wave with a supersonic Mach 2.0 transitional and turbulent boundary layer flow on an isothermal flat plate was analyzed.

DNS data provided physical insights into the transition scenario due to high intensity turbulence, that involves the three-dimensional evolution of the streamwise vortices and streaks, the generation of hairpin vortices, turbulent spots and the streak breakdown. The thermal wall conditions essentially affect three-dimensional evolutions of the quasi-streamwise vortices and the streak structures, and these differences yield inherent turbulent structures and characteristic features of the supersonic fully turbulent boundary layer.

The shock impinging simulation shows three distinct flow regions: (i) a supersonic zero pressure gradient transitional boundary layer, where low-speed streaks with successive hairpins are generated; (ii) a supersonic adverse pressure gradient region, where the interaction between the impinging shock and boundary layer structures takes place; and (iii) a supersonic zero pressure gradient region, where the turbulent flow relaxes to an equilibrium state. In the second region, the incoming boundary layer undergoes mild separation due to the adverse pressure gradient. The instantaneous velocity vectors indicate three dimensional separation features. Anisotropy invariant maps of the Reynolds stress tensor show the turbulent boundary layer undergoes the two-component axisymmetric state in the second region and reaches an equilibrium state in the third region. This shock impinging simulation with an unsteady small separation flow indicates that the turbulence enhancement is primarily associated with the adverse pressure gradient and a direct effect of the shock.

\section{Acknowledgments}

This work was partially supported by a grant-in-aid (No.21560165) from the Japanese Ministry of Education and Culture. The simulations were partly executed at the information media center of Hiroshima University and supported by associate professor Youichi Ogata.

\section{References}

(1) Guarini, S. E., Moser, R. D., Sharif, K. and Wray, A., Direct numerical simulation of a supersonic turbulent boundary layer at $M=2.5$, Journal of Fluid Mechanics, Vol. 414, 2000, pp.1-33.

(2) Gatski, T. B. and Erlebacher, G., Numerical simulation of a special evolving supersonic turbulent boundary layer, NASA TM (2002), 2002-211934.

(3) Pirozzoli, S., Grasso, F. and Gatski, T. B., Direct numerical simulation and analysis of a specially evolving supersonic turbulent boundary layer at $M=2.25$, Physics of Fluids, Vol. 16 (2004), 530.

(4) Adams, N. A., Direct simulation of the turbulent boundary layer along a compression ramp at $M=3$ and $R e_{\theta}=1685$, Journal of Fluid Mechanics, Vol. 420 (2000), pp.47-83.

(5) Pirozzoli, S. and Grasso, F., Direct numerical simulation of impinging shock wave/turbulent boundary layer interaction at $\mathrm{M}=2.25$, Physics of Fluids, Vol. 18 (2006), 065113.

(6) Deleuze, J., Structure d'une couche limite turbulente soumise á une onde de choc incidente, Ph.D. thesis, Université Aix-Marseille II (1995).

(7) Lele, S. K., Compact Finite Difference Schemes with Spectral-like Resolution, Journal of Computational Physics, Vol. 103 (1992), pp.16-42.

(8) Deng, X., Maekawa, H. and Shen, C., A class of high-order dissipative compact schemes, AIAA Paper, No. 96-1972, June 1996. 
(9) Poinsot, T. J. and Lele, S. K., Boundary conditions for direct simulations of compressive viscous flows, Journal of Computational Physics, Vol. 101 (1992), pp. 104-129.

(10) Takiguchi, T., Watanabe, D. and Maekawa, H., Application of various compact schemes to high-resolution simulations of compressible flow(in Japanese), Transaction of the Japan Society of Mechanical Engineers, Series B, Vol. 72, No. 721 (2006), pp. 104-112.

(11) Toh, S. and Itano, T., Interaction between a large scale structure and near wall structures in channel flow, Journal of Fluid Mechanics, Vol. 524 (2005), pp. 249-262.

(12) Adrian, R. J., Hairpin vortex organization in wall turbulence, Physics of Fluids, Vol. 19 (2007), No. 041301.

(13) Robinson, S. K., Coherent motions in the turbulent boundary layer, Annual Review of Fluid Mechanics, Vol. 23 (1991), pp. 601-639.

(14) Tokura, Y., Maekawa, H., and Watanabe, D., DNS of a Spatially Evolving Transitionl/Turbulent Boundary Layer at Mach 2.0 and 2.5 (submitted to AIAA Journal). Also in AIAA paper 2010-355.

(15) Morkovin, M. V., Effects of compressibility on turbulent flows, In Mécanique de la Turbulence (ed. A. Favre) CNRS (1962), pp. 367-380.

(16) Spalart, P. R., Direct simulation of a turbulent boundary layer up to $\operatorname{Re}_{\theta}=1410$, Journal of Fluid Mechanics, Vol. 187 (1988), pp. 61-98.

(17) Klebanoff, P. S., Characteristics of turbulence in a boundary layer with zero pressure gradient, NASA Report, 1247 (1955).

(18) Li, Q and Coleman, G. N., DNS of an oblique shock wave impinging upon a turbulent boundary layer, in Direct and Large-Eddy Simulation $V$, edited by R. Friedrich, B.J. Geurts, and O. Metais (Kluwer Academic, 2003), pp. 387-396.

(19) Andreopoulos, Y., Agui, J. H. and Briassulis, G., Shock wave-turbulence interactions, Annual Review of Fluid Mechanics, Vol. 32 (2000), pp. 309-345.

(20) Lumley, J. L., Computational modeling of turbulent flows, ADVANCES IN APPLIED MECHANICS, Vol. 18 (1978), pp. 123-176. 